\title{
Pathotype Grouping of Cercospora sojina Isolates on Soybean and Sensitivity to Qol Fungicides
}

\author{
Alemu Mengistu, ${ }^{1, \dagger}$ Jeffery D. Ray, ${ }^{2}$ Heather M. Kelly, ${ }^{3}$ Binbin Lin, ${ }^{3}$ Hao Yu, ${ }^{3}$ James R. Smith, ${ }^{2}$ Prakash R. Arelli, ${ }^{1}$ \\ and Nacer Bellaloui ${ }^{2}$ \\ ${ }^{1}$ USDA, Agricultural Research Service, Crop Genetics Research Unit, Jackson, TN 38301 \\ ${ }^{2}$ USDA, Agricultural Research Service, Crop Genetics Research Unit, Stoneville, MS 38776-0350 \\ ${ }^{3}$ University of Tennessee, Entomology and Plant Pathology, Jackson, TN 38301
}

\begin{abstract}
Frogeye leaf spot (FLS), caused by Cercospora sojina, is a common disease of soybean in the southern and northern United States and causes significant yield loss. The use of the current race scheme for classification for C. sojina does not take into account the range of disease severity reactions within each differential. The objective of this research was to better understand the diversity among $C$. sojina isolates through the development and use of pathogenicity groups. In this study, 83 isolates acquired from 2006 to 2009 were screened using 12 soybean (Glycine max) differentials. Disease severity on the 12 differentials ranged from 0 to 9 , where 0 is immune and 9 is very susceptible. The average severity for each isolate across differentials ranged from 1 to 7 . The 83 isolates were grouped into five pathogenicity groups (PG): PG1, PG2, PG3, PG4, and PG5, reflecting the severity grouping. Using the 12 differentials, PG1 isolates were differentiated by the lack of infection on Davis, Peking, Kent, Palmetto, Hood, CNS, Tracy, and Richland. PG2 had a range of infections on a scale of 1 to 2 on

severities from 5 to 6 . PG5 isolates caused no infection on Davis, severity of 7 on CNS, and severity of 8 on Kent, Hood, and Palmetto. The remaining seven differentials had severities of 9 . Across the geographical locations, the predominant pathotypes were PG3 and PG4 and represented $84 \%$ of the tested isolates. Azoxystrobin fungicide sensitivity tests showed that $88 \%$ of the isolates were sensitive and dominated the population, while only $6 \%$ had a high level of fungicide resistance, suggesting that FLS resistance to the QoI fungicide group was not yet completely developed and had not spread to other areas at the time when these isolates were acquired. The overall virulence profile of the isolates indicated that there was variation in disease severity, suggesting that selection of resistance for each PG may produce lines with more precisely defined interactions to specific pathotypes of $C$. sojina. This may improve the screening and selection of useful resistance genes that could be pyramided for resistance to each pathogenicity group.
\end{abstract} all differentials except on Davis; PG3 isolates had severity ranging from 3 to 4 except on Davis. PG4 isolates caused no infection on Davis, a maximum disease severity of 5 on Peking, while the rest of differentials had
Keywords: Cercospora sojina, frogeye leaf spot, pathogenicity grouping, race designation, resistance
Frogeye leaf spot (FLS), caused by the fungal pathogen Cercospora sojina, causes significant damage to soybean produced in the southern United States and is becoming important to northern states. Symptoms appear as light brown circular spots surrounded by a darkish brown to reddish circle that looks like "eye spots". As the infected leaf area reaches $50 \%$, the leaves blight, wither, and fall prematurely. Conidia, produced on conidiophore, are primary and secondary sources of inoculum and are produced on infected leaves, stems, and pods. Warm temperature and frequent rainfall promote severe disease development; and fully expanded leaves have smaller lesions compared with younger leaves (Phillips 1999). C. sojina can overwinter in soybean residue and seeds, which can be potential sources of inoculum. Cultivars with genetic resistance to FLS can be effective, with three resistance genes identified so far, including, Rcs 1 in Lincoln (Athow and Probst 1952), Rcs 2 in Kent (Athow et al.

${ }^{\dagger}$ Corresponding author: A. Mengistu; alemu.mengistu@ars.usda.gov

Funding: This study was supported in part by the Tennessee Soybean Promotion Board. This research was also funded by United States Department of Agriculture, Agricultural Research Service, project number 6066-21220013-00D.

Mention of trade names or commercial products in the publication is solely for the purpose of providing specific information and does not imply recommendation or endorsement by the U.S. Department of Agriculture. USDA is an equal opportunity provider and employer.

The author(s) declare no conflict of interest.

Accepted for publication 3 September 2019.

Modified: 17 Mar 2020.

(C) 2020 The American Phytopathological Society
1962), and Rcs3 in Davis (Phillips and Boerma 1982). The Rcs3 gene confers resistance to all known races of $C$. sojina present in the United States (Phillips and Boerma 1982; Mengistu et al. 2012) and has provided the most durable resistance against all known isolates of $C$. sojina in the United States. Another single dominant gene (reported as nonallelic to Rcs3) from the cultivar Peking was found that provided resistance against many isolates of $C$. sojina (Baker et al. 1999). The single genes conferring resistance to FLS, however, present a risk as it may be only a matter of time before they are overcome by new or rapidly evolving isolates of $C$. sojina.

For breeding programs to successfully develop resistant cultivars, knowledge of the variability and pathotype structure within the pathogen population is important. $C$. sojina isolates are variable in their aggressiveness, and new variants have shown virulence to most soybean differentials under greenhouse inoculations (A. Mengistu, unpublished), which may indicate a rapid evolution of virulence. The appearance of new virulent $C$. sojina races, such as race 2 in the late 1950s (Athow et al. 1962), races 3 and 4 in the mid-1960s (Ross 1968), and race 5 in the late 1970s (Phillips and Boerma 1981), and the associated new susceptibility of cultivars with currently deployed FLS resistance genes, have demonstrated the need to identify additional sources of genetic resistance to this pathogen. Mian et al. (2008) proposed races of $C$. sojina based on their reaction on 12 soybean differential cultivars with positive signs to indicate compatible reactions (susceptibility) and minus signs to indicate incompatible reactions (resistance). In this case, a positive sign represents disease severity ranging from low to high levels. A negative sign may mean no symptoms. Such an approach does not take into account the range of disease severity reactions within each differential. Previous studies have characterized population diversity of $C$. sojina using AFLP and simple sequence repeat (SSR) markers and showed evidence for sexual outcrossing in field populations (Bradley et al. 2012; Kim et al. 2013). However, there are no universally accepted 
methods for pathotyping isolates and no standardized soybean differentials to characterize the diversity of $C$. sojina races around the world (Grau et al. 2004). Furthermore, use of the same 12 soybean differentials has produced differing numbers of races in separate studies (Cruz and Dorrance 2009; Mian et al. 2008).

C. sojina race identification based on the current scheme suffers from two problems: (i) the variability in the aggressiveness among isolates belonging to the same race population is not differentiated, and (ii) lack of standardization among assay procedures and disease ratings has led to inflated estimates of race variability and diversity, which have made it difficult to precisely characterize $C$. sojina populations and detect new virulence types. According to Niblack et al. (2002), the value of the race scheme of Mian et al. (2008) is that it can theoretically be applied to individual races within a population, thus allowing inference of individual genotypes. However, application of race designation may not be practical since more than one race can be present in a single field at a single period of time. It is known that $C$. sojina populations vary in genetic diversity within a field (Kim et al. 2013). This variation has implications for management strategies, and a mechanism needs to be in place for documenting and discussing population changes and dynamics. To address the above concerns and to simplify the current system of $C$. sojina race designations, an alternate pathotype system, known as Pathogenicity Group, is proposed. Pathogenicity grouping of isolates incorporates the contribution of both the host and the pathogen. While the importance of a host's susceptibility to a microbe's virulence was often recognized, the existing definitions did not account for the contributions of both pathogen and host (Casadevall and Pirofski 1999). Even though resistant cultivars, crop rotation, and fungicides have historically been successful at controlling FLS, a high level of genetic diversity is known to exist in $C$. sojina, with populations likely undergoing sexual reproduction (Kim et al. 2013) and regularly creating new genetic diversity. Consequently, isolates that are resistant to quinone outside inhibitor (QoI) fungicides have emerged in many states including Alabama, Arkansas, Delaware, Illinois, Indiana, Iowa, Kentucky, Louisiana, Mississippi, Missouri, North Carolina, Ohio, Tennessee, and Virginia (Bradley et al. 2013; Zhang et al. 2012a) since 2010, thus reducing FLS control options (Zhang et al. 2018). This fungicide resistance mechanism is based on a single point mutation, resulting in a single change in amino acids from glycine to alanine (G143A) found in the mitochondrial cytochrome $b$ gene (CYTB) (Gisi et al. 2002). Better understanding of the variability of $C$. sojina in soybean fields is therefore important to determine the dynamics of fungicide resistance in populations as well as to develop fungicide chemistry to control specific groups of pathotypes rather than races. This would require following established baseline sensitivities to QoI fungicides to be able to detect shifts in sensitivity (Zhang et al. 2012b). The purpose of this study, therefore, was to evaluate the range of variability in virulence among isolates of $C$. sojina based on qualitative disease severity ratings using soybean differentials and to identify isolate sensitivity to the QoI fungicides. A new pathotype system, based on pathogenicity groups, is proposed and evaluated as an alternative to the prior race scheme of Mian et al. (2008).

\section{Materials and Methods}

Disease severity of the differentials. Eighty-three isolates were acquired from various geographical regions in 2006 to 2009: seven from Alabama, five from Arkansas, eight from Brazil, three from China, one from Florida, 15 from Georgia, two from Illinois, two from Iowa, three from Louisiana, eight from Mississippi, two from South Carolina, 26 from Tennessee, and one from Wisconsin. Disease severity was determined using a set of 12 soybean differentials: Davis (Caviness and Walters 1966), Peking (Gizlice et al. 1994), Kent (Probst and Athow 1964), CNS (Gizlice et al. 1994), Palmetto (Gizlice et al. 1994), Tracy (Hartwig 1974), Lincoln (Weiss 1953a), S-100 (Gizlice et al. 1994), Richland (Gizlice et al. 1994), Blackhawk (Weiss 1953b), Hood (Johnson 1960), and Lee (Johnson 1958). Monoconidial isolates from each of the 83 isolates were generated and culture plates were flooded with sterile deionized water.
The surface of the culture was rubbed manually to dislodge conidia.

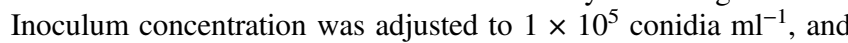
polysorbate-20 (Tween 20, a viscous liquid, Sigma Aldrich, St. Louis, MO) was mixed as a spreader with the suspension $(0.03 \%$ $\mathrm{vol} / \mathrm{vol})$. Eight soybean seedlings with two plants per replication per differential were used for inoculation at the V2-V3 growth stage (Fehr and Caviness 1977) by atomizing the inoculum with approximately $125 \mathrm{ml}$ of water to cover the entire plant leaf surface. Inoculated plants were then placed in a dew chamber with $100 \%$ humidity at $24^{\circ} \mathrm{C}$ for $24 \mathrm{~h}$ in the dark and then moved to a growth chamber where the temperature was maintained at $24^{\circ} \mathrm{C}$ with a 12 -h photoperiod. Disease severity ratings were taken 14 days after inoculation using a 0 to 9 scale (Wallace 1982) in which $0=$ no disease and $9 \geq 90 \%$ of leaf area diseased. All leaves on each plant were examined for lesions, and the average rating was recorded. Plants were further evaluated for reaction to $C$. sojina at 21 and 28 days after inoculation to verify if an increase in disease severity had occurred; and the maximum severity rating was used in the data analysis.

In vitro fungicide sensitivity tests. A total of 64 of the 83 FLS isolates were tested for fungicide sensitivity. Isolates of $C$. sojina were cultured on V-8 agar plates at $25^{\circ} \mathrm{C}$ in the dark for 10 days. Mycelial disks were removed from the margins of actively growing cultures using a sterile cork-borer $(5 \mathrm{~mm}$ in diameter) and transferred to the center of fresh potato dextrose agar (PDA) (Difco Laboratories, Detroit, MI) plates for radial growth measurements. PDA media was amended with azoxystrobin at a discriminatory dose of $0.001 \mathrm{~g} / \mathrm{ml}$, as suggested by Zhang et al. (2012b). Both fungicideamended and nonamended plates were supplemented with salicylhydroxamic acid (SHAM) at $0.06 \mathrm{~g} / \mathrm{ml}$ dissolved in methanol, to prevent the alternative oxidase respiratory pathway in QoIsensitive conidia from being used to overcome the inhibitory effect of the fungicide once plated onto the media (Vanlerberghe 2013). The average colony diameter was then recorded by measuring two cross lines of radial growth at a right angle on the plates after 3 weeks of incubation in the dark at $25^{\circ} \mathrm{C}$. The percent growth inhibition was then calculated using the formula ([colony diameter on fungicide-free medium - $5 \mathrm{~mm}$ ] - [colony diameter on azoxystrobin-amended medium $-5 \mathrm{~mm}]$ )/(colony diameter on fungicide-free medium $-5 \mathrm{~mm}) \times 100$ (Ishii et al. 2007). In addition, based on the growth inhibition rate, isolates were divided into various levels of sensitivity (Villani and Cox 2014) with different ranges (0 to $50 \%$ growth inhibition indicating high resistance responses, 51 to $71 \%$ growth inhibition indicating moderate resistance responses, and 72 to $100 \%$ growth inhibition indicating sensitive response). For each isolate, three replicate plates were evaluated. This experiment was repeated twice.

Data analysis. A hierarchical cluster analysis was initially used to group the isolates and the differentials. The Ward's method was used for the analysis of variance approach for the evaluation of distances between clusters. Ward's method attempts to minimize the sum of squares of any two hypothetical clusters that can be formed at each step (Ward 1963). $P$ values were used to choose the appropriate number of clusters. ANOVA was used to test the differences in severities among groups. The cluster analysis, relative response to differentials, and PG analysis were conducted using the 0 to 9 scale. However, in order to simplify the large data set of host-pathogen interactions, the 0 to 9 scale was converted to 0 to 5 scale for analysis, where $0=0$ (immune); 1 to $2=1$ (resistant); 3 to $4=2$ (moderately resistant); 5 to $6=$ 3 (moderately susceptible); 7 to $8=4$ (susceptible); and $9=5$ (very susceptible). Resistance Index $=$ the number of isolates to which the differential is resistant $\div 83$ (total number of isolates tested) $\times 100$ ) was computed. The data were converted back to 0 to 9 scale for presentation.

\section{Results}

Severity rating and grouping of isolates. Isolate and differential combinations are shown with their corresponding colors representing severity classes based on 0 to 5 scale (Fig. 1) derived from the measurement scale of 0 to 9 . Out of the 996 possible interactions (12 differentials $* 83$ isolates), $52(5 \%)$ of the interactions 
were from isolates with a 0 rating (immune), 281 (28\%) of the interactions were from isolates with a 1 severity rating (resistant), $253(25 \%)$ of the interactions were from isolates with a 2 rating (moderately resistant), $219(22 \%)$ of the interactions were from isolates that had a 3 rating (moderately susceptible), $166(17 \%)$ of the interactions were from isolates that had a rating of 4 (susceptible), and $25(3 \%)$ of the interactions had a rating of 5 (very susceptible).

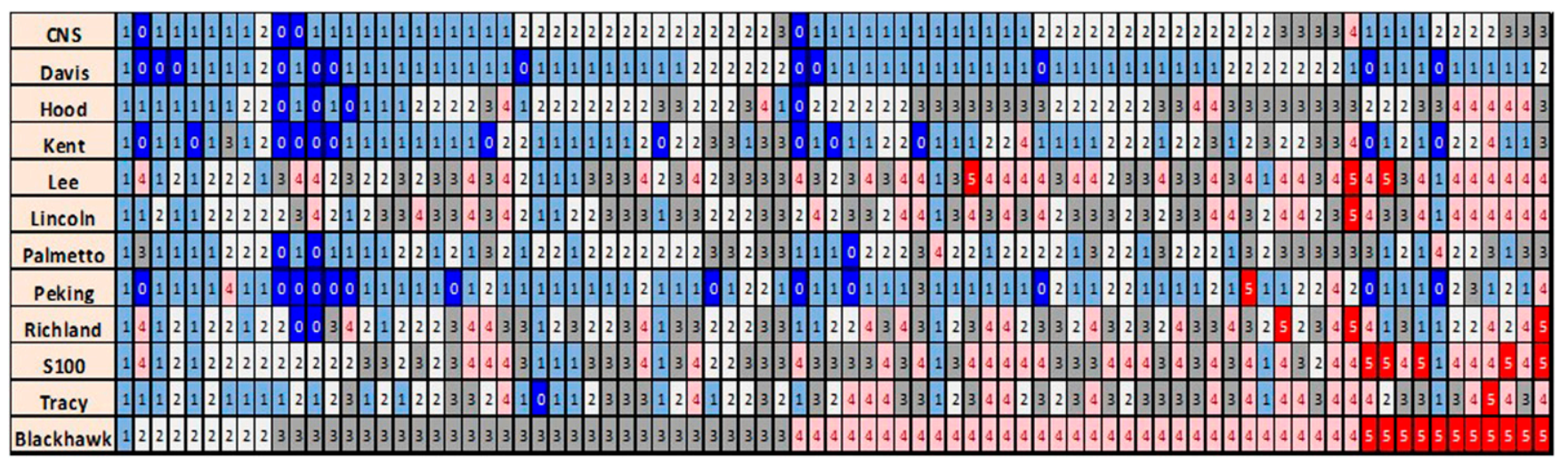

Fig. 1. The average scaling of 0 to 9 converted to 0 to 5 , where resistance classification for Cercospora sojina is: 0,0 (immune); 1 to 2,1 (resistant); 3 to 4,2 (moderately resistant); 5 to 6,3 (moderately susceptible); 7 to 8,4 (susceptible); 9,5 (very susceptible), respectively, based on the 12 soybean differentials and the number of interactions.

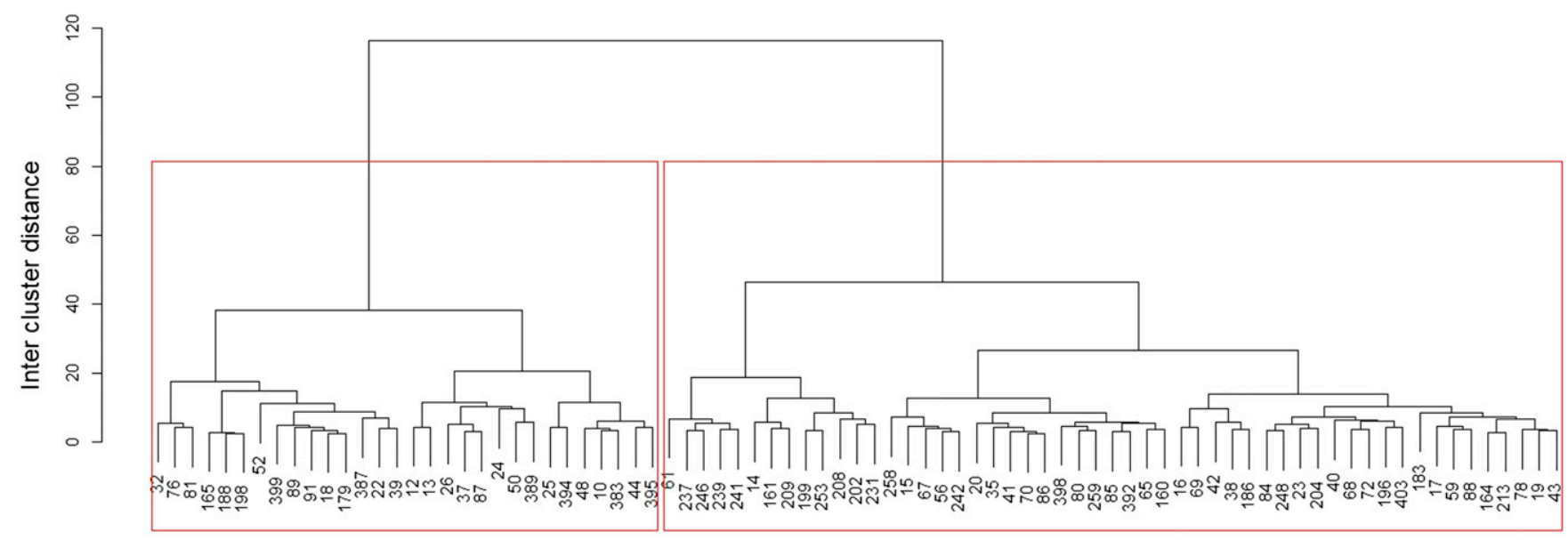

Isolates

Fig. 2. Cluster Dendrogram of 83 isolates of Cercospora sojina. The isolates in the same rectangle belong to the same pathogenicity group.

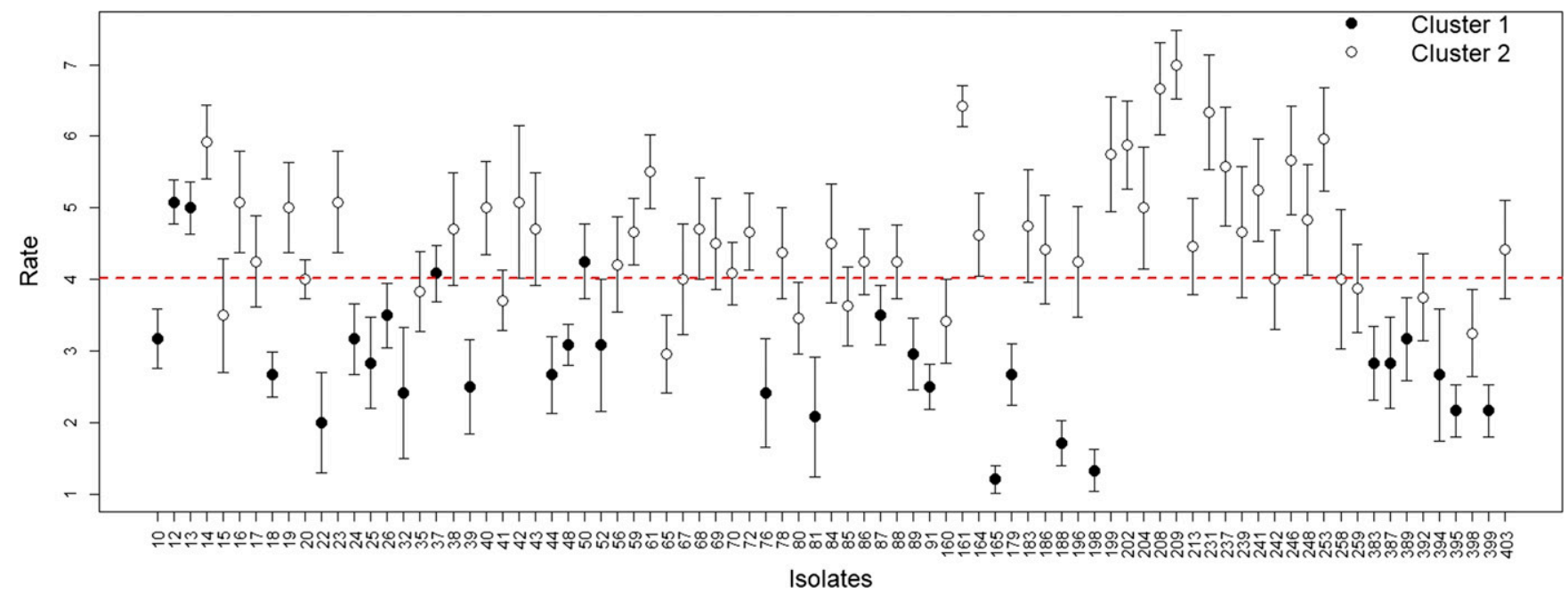

Fig. 3. Mean severity of each isolate of Cercospora sojina. The shaded circles indicate mean severity of Cluster 1, whereas the open circles indicate mean severity of Cluster 2. The vertical lines indicate standard errors of each mean. 
The disease severity ( 0 to 9 scale) of the 83 isolates of $C$. sojina was initially used to group the isolates into clusters of similarity based on the response across all 12 differentials (Fig. 2). The isolates were grouped into two major clusters with two subgroups within each major cluster. The group of isolates within the first cluster had isolates with low severity values $(<4)$, but also included 4 isolates with higher severity values $(>4)$ based on the 0 to 9 scale (Fig. 2 ). The second cluster consists of isolates with higher severity values than in cluster 1 but with a much broader range of severity values (3 to 7) (Fig. 2). The mean severity value across each isolate within each cluster is further shown in Figure 3, where the majority of isolates in cluster 1 have lower severity values below the mean (4) than for the isolates in cluster 2 .

The trend of disease increase was shown when all of the 83 isolates were plotted by their severity values across the 12 differentials (Fig. 4). The analysis of variance (not shown) indicated that both isolates and differentials significantly affected the disease severity rating $(P \leq 0.01)$. The degree of infection across the 12 differentials provided data that showed various levels of aggressiveness of the isolates. Isolate 209, for example, had the highest overall mean severity of 7, and isolate 165 had the lowest mean severity of 1.2 across the 12 differentials (Fig. 4). For each isolate, the severity varied by each differential and in some cases remained the same across differentials. The mean severity value across differentials was 4 .

Figure 5 shows the relative response of each differential to the 83 isolates relative to the average score of each isolate over all the 12 differentials. In this case, Blackhawk was more susceptible to all isolates than the average score across all differentials. In contrast, Davis was more resistant to most isolates than the average over all differentials. The responses of the other differentials ranged between those of Blackhawk and Davis.

Davis and Peking are two of the most resistant differentials, based on their cumulative responses to the 83 isolates. However, comparison of specific isolate responses of these two differentials highlights important potential genetic differences between them. While the overall relative responses of these two differentials were similar to most isolates, there were two isolates for which Peking was considerably more susceptible than was Davis (isolates 14 and 24; two highest data points for Peking). Likewise, Blackhawk and Lee, two of the most susceptible differentials, showed considerable difference in their reactions to the five isolates below the average resistance line. Again, there are likely genetic differences between these cultivars underlying these disease ratings. In this study, the reaction of these differentials spanned a resistance index ranging from 1 to $99 \%$ (Table 1).
In vitro fungicide sensitivity tests. Sensitivities of fungal isolates to azoxystrobin were examined based on mycelial growth on fungicide-amended PDA plates (Table 2). Three levels of sensitivity were found in these isolates, where 0 to $50 \%$ growth inhibition was considered insensitive (resistant) to azoxystrobin, 51 to $71 \%$ indicated moderately resistant, and $>71 \%$ indicated sensitivity (susceptible). Four isolates (TN188, TN198, TN392, and TN399) were considered to be insensitive. Four isolates (TN14, TN19, TN199, and TN208) were considered to be moderately insensitive (51 and $71 \%$ growth inhibition). The majority of the isolates (56 isolates) were sensitive (Table 2). Among the four highly resistant isolates to azoxystrobin, one was PG1, two were PG2, and one was PG3. Of the moderately resistant isolates to azoxystrobin, three were PG4 and one was PG5. The rest of the isolates (56) were distributed over PG1 through PG5, all being sensitive to azoxystrobin. When all the isolates were grouped to the 12 differentials, the number of

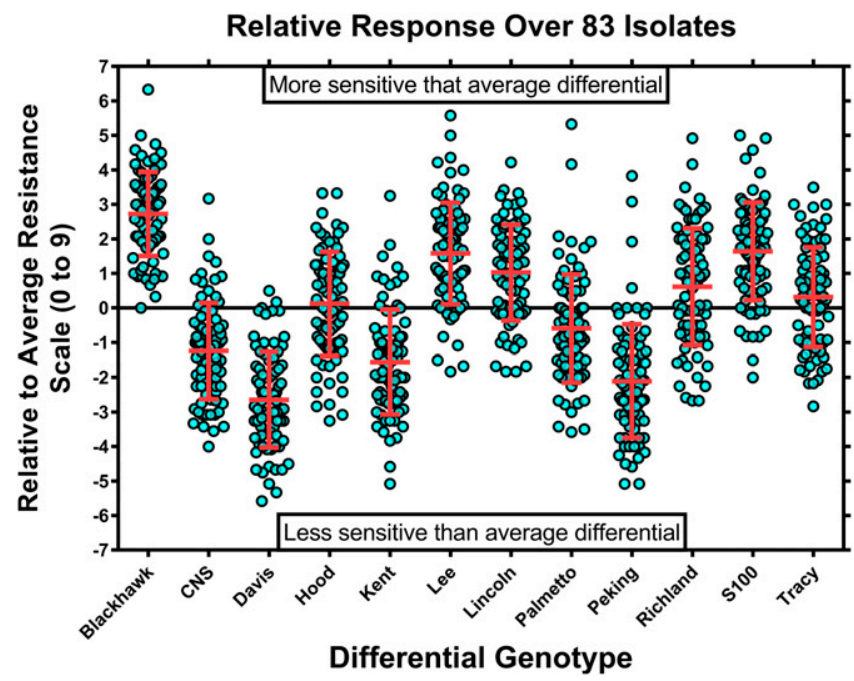

Fig. 5. Relative response of the 12 soybean differentials (in alphabetical order) to 83 Cercospora sojina isolates relative to the average score ( 0 to 9 scale) of each isolate over all 12 differentials showing individual data points. Note that the horizontal red lines in the middle indicate the means, and the vertical red lines indicate standard errors of each mean. Positive numbers on the $y$-axis indicate that the specific differential was more sensitive than the average of the 12 differentials, whereas negative numbers indicate it was less sensitive.

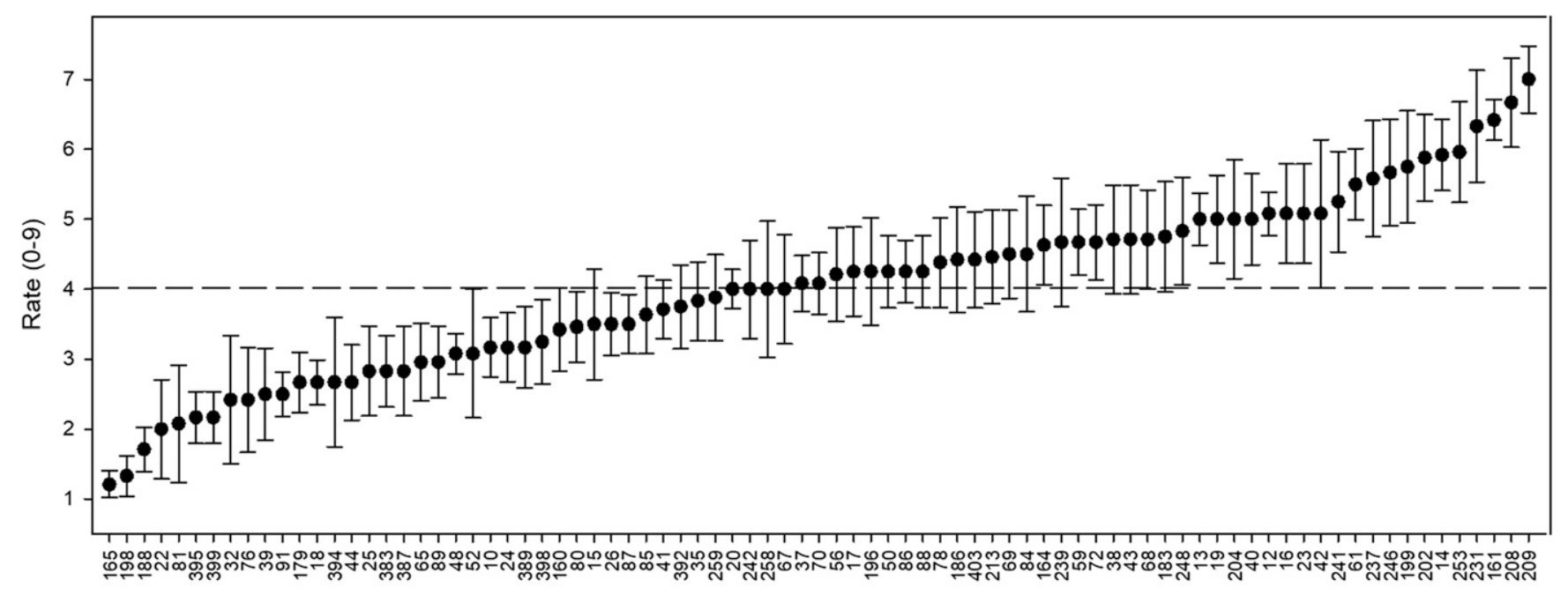

Isolates

Fig. 4. Mean severity responses of the 83 isolates of Cercospora sojina across 12 soybean differentials ordered by isolate severity. The dots represent mean severity and the vertical lines indicate standard errors of each mean. 
isolates for each pathogenicity group (PG) was determined (Table 3). PG1 isolates were differentiated by the lack of infection on Davis, Peking, Kent, Palmetto, Hood, CNS, Tracy, and Richland. PG2 had a range of infections on a scale of 1 to 2 on all differentials except on Davis; PG3 isolates had severity ranging from 3 to 4 except on Davis. PG4 isolates caused no infection on Davis, a maximum disease severity of 5 on Peking, while the rest of differentials had severities from 5 to 6 . PG5 isolates caused no infection on Davis. However, CNS had a maximum severity of 7, while Palmetto, Hood, and Lee had a maximum severity of 8 . The rest of the differentials had a maximum severity of 9. PG2 and PG3 isolates caused a hypersensitivity (resistant reaction) on Davis, but the reaction was rated as $1-2$ and $3-4$, similar for the rest of the differentials. Davis tended to show varying levels of minute necrotic leaf spots of less than $0.1 \mathrm{~cm}$, characteristic of the hypersensitive response that often leads to cell death. Such a response is an indication often associated with resistance to pathogen infection.

The number of isolates that caused severe infection were $0,1,2,3$, and 4 for Davis, CNS, Palmetto, Kent, and Peking, respectively. Blackhawk had infections across all PGs with the highest number of isolates (44) in the severity ranges of 7 to 9 , followed by Lee (34) and S-100 (33), all in PG 5 (Table 3). Not all of the isolates within each PG infected all the differentials. Some of the isolates caused severity in one or more differentials. Based on the 12 differentials, the disease severity classification was designated as "Pathogenicity Groups" PG1 to PG5. Pathogenicity grouping of isolates takes into consideration the response of each differential for each isolate. The relative proposed pathotypes (PG1 to PG5) of C. sojina isolates based on a 0 to 9 scale and their reactions on the 12 soybean differential cultivars is presented in Figure 6. Spots on the leaf represent compatible reactions (susceptibility) and absence of leaf spots represents incompatible reactions (resistance).

Isolates of $C$. sojina from the United States, China, and Brazil were differentiated into five pathogenicity groups based on the mean of maximum disease severity of FLS symptoms on infected leaves (Table 4). Of the 83 isolates, 67 were collected in the southern United States. Of these, 52 (78\%) isolates were grouped into PG3 and PG4 (Table 4). Isolates in PG2, PG3, and PG4 were common and were found in most locations. On the other hand, PG1 and PG5 were not detected from any other locations except in Tennessee.

\section{Discussion}

The proposed classification of $C$. sojina isolates into pathogenicity groups has at least four important uses: (1) as a mechanism for assessing and documenting changes in prevalent field isolates over time; (2) for use by plant pathologists and soybean breeders for producing resistant cultivars/germplasms; (3) as a means of describing the PG classification used for screening; and (4) as an aid to making

Table 1. Resistance index of 12 soybean differential cultivars to 83 isolates of Cercospora sojina and their selection criteria

\begin{tabular}{llcccc}
\hline $\begin{array}{l}\text { Selected } \\
\text { differential } \\
\text { cultivars }\end{array}$ & $\begin{array}{l}\text { Selection } \\
\text { criterion }\end{array}$ & Positive & Negative & $\begin{array}{c}\text { Positive/ } \\
\text { Negative } \\
\text { ratio }^{\mathbf{a}}\end{array}$ & $\begin{array}{c}\text { Resistance } \\
\text { index }^{\mathbf{b}}\end{array}$ \\
\hline Davis & Rcs3 gene & 1 & 83 & 0.01 & 100 \\
Peking & Ancestor & 3 & 80 & 0.04 & 96 \\
Kent & Rcs2 gene & 10 & 73 & 0.1 & 88 \\
CNS & Ancestor & 15 & 68 & 0.2 & 82 \\
Palmetto & Ancestor & 19 & 64 & 0.3 & 77 \\
Hood & Cultivars & 41 & 42 & 1 & 51 \\
Richland & Ancestor & 48 & 35 & 1.4 & 42 \\
Tracy & Ancestor & 49 & 34 & 1.4 & 41 \\
Lincoln & Rcs1 gene & 64 & 19 & 3.4 & 23 \\
S-100 & Ancestor & 70 & 13 & 5.4 & 16 \\
Lee & Cultivars & 72 & 11 & 6.5 & 13 \\
Blackhawk & Susceptible & 82 & 1 & 82 & 1 \\
\hline
\end{tabular}

${ }^{a}$ Ratio indicates positive infection over negative infection.

${ }^{b}$ Percent resistance index $=$ number of isolates to which the differential is resistant/83 (total number of isolates tested) $\times 100$. management recommendations to growers. The system could be widely adopted by the soybean industry, academia, and extension workers as a standardized method for soybean screening/reporting frogeye resistance. In this study, we are proposing the broader term of pathogenicity group rather than race for a better understanding of the genetic basis for pathogenicity, pathogen variability, and host-pathogen interaction. It also avoids the implication of genetic uniformity or predictability in contrast to the way the current race scheme is being used.

Three of the 12 differentials conditioning single gene resistance: Lincoln, Kent, and Davis are known sources for the Rcs1, Rcs2, and Rcs 3 resistance genes, respectively. Interestingly, Kent is derived from Lincoln, but has Rcs2, not Rcs 1. Kent's other parent, 'Ogden' (Weiss 1953c), may be the source of Rcs2. Peking, CNS, Palmetto, Richland, Tracy, and S-100 were added because they represent ancestors to most current North American public cultivars

Table 2. Reaction of Cercospora sojina isolates from broad geographical regions to azoxystrobin fungicide at $1 \mathrm{mg} / \mathrm{ml}^{\mathrm{a}}$

\begin{tabular}{cc}
\hline Growth inhibition (\%) & Individual TN TN$^{\mathbf{b}}$ isolates \\
\hline 0 to $50 \%$ (insensitive response) & TN188, TN198, TN392, TN399 \\
(resistance) & \\
51 to $71 \%$ (moderately insensitive) & TN14, TN19, TN199, TN208 \\
(moderately resistance responses) & \\
72 to $100 \%$ (sensitive response) & TN10, TN15, TN16, TN17, TN 19 \\
(susceptible) & TN20, TN22, TN23, TN32, TN37, \\
& TN38, TN39, TN40, TN41, TN42, \\
& TN43, TN52, TN56, TN65, TN67, \\
& TN68, TN69, TN70, TN72, TN76, \\
& TN80, TN81, TN84, TN85, TN86, \\
& TN88, TN89, TN91, TN160, \\
& TN161, TN164, TN165, TN179, \\
& TN183, TN186, TN196, TN202, \\
& TN204, TN209, TN213, TN231, \\
& TN237,TN241, TN242, \\
& TN246,TN256, TN383, TN387, \\
& TN394, TN398, TN403
\end{tabular}

a Growth inhibition (\%) of 3-week culture growth was calculated as ([colony diameter on fungicide-free medium - $5 \mathrm{~mm}$ ] - [colony diameter on azoxystrobin-amended medium $-5 \mathrm{~mm}]) /($ colony diameter on fungicide-free medium $-5 \mathrm{~mm}) \times 100$.

${ }^{\mathrm{b}}$ Refers to Tennessee designated name for isolates.

Table 3. Mean severity values (parentheses) for frogeye based on the $0-9$ scale for the isolate soybean differential interaction and their corresponding number of isolates identified in each pathogenicity group (PG) differential combination

\begin{tabular}{lccccc}
\hline & \multicolumn{5}{c}{ Number of isolates within each PG } \\
\cline { 2 - 6 } Differentials & PG1 (0) & PG2 (1-2) & PG3 (3-4) & PG4 (5-6) & PG5 (7-9) \\
\hline Davis & 12 & $56^{\mathrm{a}}$ & $15^{\mathrm{a}}$ & 0 & 0 \\
Peking & 13 & 51 & 13 & 2 & 4 \\
Kent & 13 & 36 & 21 & 10 & 3 \\
Palmetto & 3 & 24 & 34 & 20 & 2 \\
Hood & 4 & 14 & 31 & 24 & 10 \\
CNS & 4 & 36 & 34 & 8 & 1 \\
Tracy & 1 & 20 & 19 & 24 & 19 \\
Richland & 2 & 13 & 27 & 22 & 19 \\
Lincoln b & - & 11 & 21 & 28 & 23 \\
S-100 & - & 10 & 15 & 25 & 33 \\
Lee $^{\text {b }}$ & - & 10 & 11 & 28 & 34 \\
Blackhawk $^{\mathrm{b}}$ & - & $+\mathrm{c}$ & 9 & 30 & 44 \\
\hline
\end{tabular}

${ }^{a}$ Indicates that Davis had a hypersensitive reaction to these isolates.

${ }^{b}$ Indicates that Lincoln, S-100, Lee, and Blackhawk had a rating of 1 to 2 and didn't belong in PG1. The average scaling of 0 to 9, where resistance classification of 0 is immune; 1 to 2 is resistant; 3 to 4 moderately resistant; 5 to 6 moderately susceptible; 7 to 9 is grouped as susceptible based on the 12 differentials and the number of interactions.

c $¥$ indicates that Blackhawk had a rating of 3-4 and didn’t belong in PG2. 
(Mian et al. 2008). Hood was added as a potential differential (Mian et al. 2008). Blackhawk was included because of its universal susceptibility to FLS. Richland is a parent of Blackhawk (Richland $\times$ Mukden), but is not nearly as susceptible as Blackhawk. The final differential, Lee, adds significant variability to the group (Gizlice et al. 1994). This is in agreement with what Mian et al. (2008) reported. As a group, these differentials account for over $50 \%$ of the variability that occurred due to phenotypic diversity in 258 North
American public cultivars released between 1947 and 1988 (Gizlice et al. 1994). Hence, as a group, they represent considerable genotypic variability. Even though the overall relative responses for Davis and Kent were similar for most isolates, Peking was considerably more susceptible than was Davis to two of the isolates (14 and 24). These differential responses may indicate an underlying genetic difference between Davis and Peking that should be further investigated. Such difference may also be due to $C$. sojina undergoing sexual

\begin{tabular}{|c|c|c|c|c|c|}
\hline Differentials & PGl (0) & PG2 (1-2) & PG3 (3-4) & PG4 (5-6) & PG5 (7-9) \\
\hline Davis & & (HR) & (HR & & \\
\hline Peking & & & & & \\
\hline Kent & & & & & \\
\hline $\mathrm{CNS}$ & & & & & \\
\hline Palmetto & & & & & \\
\hline Hood & & & & & \\
\hline Tracy & & & & & \\
\hline Richland & & & & & \\
\hline Lincoln & & & & & \\
\hline Lee & & & & & \\
\hline$\$ 100$ & & & & & \\
\hline Blackhawk & & & & & \\
\hline
\end{tabular}

Fig. 6. Relative proposed pathotypes (PG1 to PG5) of Cercospora sojina isolates based on 0 to 9 scale and their reaction on six soybean differential cultivars. Spots on the leaves represent compatible reactions (susceptibility) and absence of leaf spots represent incompatible reactions (resistance). 
reproduction (Kim et al. 2013; Shrestha et al. 2017). In many plantpathogenic fungi, sexual reproduction is a key mechanism through which genetic diversity is produced, and often allows fungal pathogens to overcome host resistance by generating more fit genotypes through recombination (Glass and Kuldau 1992).

Analysis of variance showed that there were significant isolate, differential, and isolate-by-differential interactions, indicating that both isolates and differentials were significantly affecting severity ratings. The degree of infection on the 12 differentials varied by isolate aggressiveness within each pathotype, indicating the benefit of selecting for resistance to known pathotypes. Even though cluster analysis was able to place the isolates into two major groups, some isolates from specific PG groups were detected in either cluster 1 or 2. Thus, the hierarchical classification used in the dendrogram did not always manifest a direct effect on host pathogen interactions, whereas the pathogenicity grouping classification did. The conversion of disease severity from 0 -to- 9 to 0 -to- 5 placed large amounts of host-pathogen interaction data into the same plane and provided a more interpretive and useful grouping than the cluster analysis. Such scaling produced classes that are meaningful and distinct as defined by the disease severity scales. The pathogenicity grouping approach allowed the classification of the most virulent pathotype that infected all genotypes (PG5) except Davis; pathotypes that had no infection on Davis but moderate infection on Peking (PG4); pathotypes with a hypersensitive reaction to Davis but with less than moderate reaction to the rest of the differentials (PG3); pathotypes with low virulence (except for Davis with a hypersensitive reaction) (PG2); and pathotypes with no infection on eight of the differentials except on Blackhawk, Lincoln, S-100, and Lee (PG1). The 12 differentials selected by Mian et al. (2008) were retained because of the range of their resistance index relative response to the 83 isolates and the extent of their contribution to the ancestry of the major North American public cultivars.

Of the 11 combined isolates from China and Brazil, nine were in PG3, indicating that they were less diverse than originally thought. The groups PG1 and PG5 were not detected from any other locations except in Tennessee. This may well be due to the unequal number of isolate samples taken from each geographical origin. However, when isolates from Georgia and Tennessee, where sample number was comparable, were compared, the isolates from Tennessee still showed more diversity than those from Georgia. Because of the small sample size and the nonrandom nature of the collection of these isolates from several geographical regions, no conclusions about relative frequencies of PG types can be drawn, although the fact that all isolates from PG1 to PG5 were present only in Tennessee may suggest the existence of wide variability of isolates. This is supported by the findings of Shrestha et al. (2017) that both mating type alleles (MAT1-1-1 and MAT1-2) were detected in Jackson and Milan, TN just from single lesion isolates, suggesting sexual recombination. The presence of sexual recombination also suggested it had a role in the epidemiology of field populations and is responsible for the existence of wide variability of $C$. sojina isolates. Even though there is no indication of the relationship between the geographical regions and pathogen grouping, future breeding efforts may need to focus on pathogenicity group rather than on using isolates with specific race designations. In future studies, it may be possible that additional pathogenicity groups could be identified if additional differential genotypic variability was employed in the form of such unique ancestors as Madarin (Ottawa), AK (Harrow), PI 54610, Dunfield, and Mukden. According to Gizlice et al. (1994), these soybean genotypes may account for almost an additional $25 \%$ of the North American soybean cultivar genetic base. Furthermore, Mukden, as a parent of Blackhawk, could be highly susceptible but still different than the highly susceptible Blackhawk.

The $C$. sojina isolates acquired from wide geographical locations in 2006, 2007, and 2009 were evaluated using a mycelial growth assay and were found to be in three different levels of sensitivity to azoxystrobin. In general, the sensitive isolates dominated the population (88\%), which can be expected as 26 of the 64 isolates evaluated were previously used in Zhang et al. (2012b) as the baseline group which determined the $\mathrm{EC}_{50}$ to azoxystrobin, pyraclostrobin, and trifloxystrobin. While only 5\% of the isolates from 2007 and 2009 had a high resistance response, this is possible as selection pressure could have been exerted on these isolates since QoI fungicides were registered on soybean prior to the acquired dates. This suggests that FLS resistance may not have completely developed and had not spread to other areas at the time when some of these isolates were initially acquired in 2006. The presence of highly resistant and moderately resistant isolates, however, predates the findings of Zhang et al. (2012a) that FLS resistance was first reported in 2010 in West Tennessee. Our results showed resistance had already begun to develop but had not been investigated and reported until 2010. Similarly, the abundance of isolates with moderate resistance responses was low, at only $6 \%$ out of the total number of isolates tested. The low abundance of the moderately resistant isolates may suggest that other unidentified genetic factors may better explain the partial resistance response in some isolates or the less common point mutations that have been reported to convey qualitative resistance (Fisher and Meunier 2008; Villani and Cox 2014). Additionally, the discriminatory dose used for these evaluations states that resistant isolates had at least $50 \%$ of conidia germinate on $0.001 \mathrm{~g} / \mathrm{ml}$ of azoxystrobin, and the moderate resistance responses in this study fall under that $50 \%$ and could be considered sensitive under initial description of the discriminatory dose (Zhang 2012). Furthermore, Mengistu et al. (2014) reported that three out of four QoI-resistant strains collected in 2010 was not indicative of the amount of QoI resistance present in the field. It was only an indication that QoI-resistant strains were present. Results from QoI sensitivity testing, using bulked samples of $C$. sojina from the Milan Research and Education Center in 2012, also revealed only 0 to $15 \%$ of conidia were resistant to QoI fungicides (Zhang et al. 2018). Hence, it can be speculated that while QoI-resistant strains were present since 2007, they were not the dominant strain present.

This study has identified 5 pathogenicity groups from 83 isolates using 12 differentials, designated as PG1 through PG5, to represent the virulence diversity present in isolates from various geographical regions. Some of these $C$. sojina isolates have previously been deposited in the American Type Culture Collection (Mian et al. 2008) but are also maintained currently as a working collection by the senior author (A. Mengistu, USDA, Jackson, TN). The PG classification may allow

Table 4. Classification of isolates of Cercospora sojina from different countries into pathogenicity groups based on responses from 12 soybean differentials

\begin{tabular}{|c|c|c|c|c|c|c|}
\hline \multirow[b]{2}{*}{ Origin } & \multirow{2}{*}{$\begin{array}{c}\text { Total number } \\
\text { of isolates }^{\mathbf{a}}\end{array}$} & \multicolumn{5}{|c|}{$\begin{array}{c}\text { Number of isolates in } \\
\text { pathogenicity group (PG)b }\end{array}$} \\
\hline & & 1 & 2 & 3 & 4 & 5 \\
\hline$\overline{\text { Alabama }}$ & 7 & & 2 & 3 & 2 & \\
\hline Arkansas & 5 & & & 5 & & \\
\hline Brazil & 8 & & 1 & 5 & 2 & \\
\hline China & 3 & & & 3 & & \\
\hline Florida & 1 & & & & 1 & \\
\hline Georgia & 15 & & 2 & 8 & 5 & \\
\hline Illinois & 2 & & & 2 & & \\
\hline Iowa & 2 & & & 2 & & \\
\hline Louisiana & 3 & & 1 & 1 & 1 & \\
\hline Mississippi & 9 & & 1 & 5 & 3 & \\
\hline South Carolina & 1 & & & 1 & & \\
\hline Tennessee & 26 & 1 & 2 & 7 & 14 & 2 \\
\hline Wisconsin & 1 & & & 1 & & \\
\hline Total & 83 & 1 & 9 & 43 & 28 & 2 \\
\hline
\end{tabular}

a Total number of isolates acquired from each location.

${ }^{b}$ Isolates represented by each PG group from each location. PG1 isolates caused infection on Blackhawk, Lincoln, Lee, and S100 $(>0<1)$; PG2 had a severity of 1 to 2 on all differentials except Davis; PG3 isolates had disease severity from 3 to 4 except for Davis; PG4 isolates caused no infection on Davis, but Peking had a maximum disease severity of 5, while the rest of the differentials had severity from 5 to 6 . PG5 isolates caused no infection on Davis, but the rest of the differentials had a severity ranging from 7 to 9 . 
a better understanding of the genetics of plant-pathogen interactions and will enable approaches to achieve sustainable disease resistance.

\section{Acknowledgments}

We thank Jason Deffenbaugh, Jamie Jordan, Tara Sydboten, and Amber Wesley for their efforts in this project.

\section{Literature Cited}

Athow, K. L., and Probst, A. H. 1952. The inheritance of resistance to frogeye leaf spot of soybeans. Phytopathology 42:660-662.

Athow, K. L., Probst, A. H., Kurtzman, C. P., and Laviolette, F. A. 1962. A newly identified physiological race of Cercospora sojina on soybean. Phytopathology 52:712-714.

Baker, W. A., Weaver, D. B., Qiu, J., and Pace, P. F. 1999. Genetic analysis of frogeye leaf spot resistance in PI54610 and Peking soybean. Crop Sci. 39:1021-1025.

Bradley, A. C., Zhang, G., Chapara, V., Ming, R., Zeng, F., Young, H. K., and Newman, N. 2013. Update on detection and management of QoI fungicide resistant Cercospora sojina, the causal agent of frogeye leaf spot in soybean. Page 9 in: Proc. 2013 SSDW Meeting, Pensacola, FL.

Bradley, C., Wood, A., Zhang, G., Murray, J., Phillips, D., and Ming, R. 2012. Genetic diversity of Cercospora sojina revealed by amplified fragment length polymorphism markers. Can. J. Plant Pathol. 34:410-416.

Casadevall, A., and Pirofski, L. A. 1999. Host-pathogen interactions: Redefining the basic concepts of virulence and pathogenicity. Infect. Immun. 67: 3703-3713.

Caviness, C. E., and Walters, H. J. 1966. Registration of Davis soybeans (Reg. No. 56). Crop Sci. 6:502.

Cruz, C., and Dorrance, A. 2009. Characterization and survival of Cercospora sojina in Ohio. Plant Health Prog. 10:17.

Fehr, W. R., and Caviness, C. E. 1977. Stages of soybean development. Spec. Rep. 80. Iowa Cooperative Ext. Serv., Ames, IA.

Fisher, N., and Meunier, B. 2008. Molecular basis of resistance to cytochrome bc1 inhibitors. FEMS Yeast Res. 8:183-192.

Gisi, U. H., Sierotzki, H., Cook, A., and McCaffery, A. 2002. Mechanisms influencing the evolution of resistance to Qo inhibitor fungicides. Pest Manag. Sci. 58:859-867.

Gizlice, Z., Carter, T. E., and Burton, J. W. 1994. Genetic base of North American public soybean cultivars released between 1947 and 1988. Crop Sci. 34: 1143-1151.

Glass, L. N., and Kuldau, G. A. 1992. Mating type and vegetative incompatibility in filamentous ascomycetes. Annu. Rev. Phytopathol. 30:201-224.

Grau, C. R., Dorrance, A. E., Bond, J., and Russin, J. S. 2004. Fungal diseases. Soybeans. Pages 679-763 in: Improvement, Production, and Uses, 3rd ed. American Society of Agronomy, Madison, WI.

Hartwig, E. E. 1974. Registration of Tracy Soybeans (Reg. No. 105). Crop Sci. 14:777.

Ishii, H., Yano, K., Date, H., Furuta, A., Sagehashi, Y., Yamaguchi, T., et al. 2007. Molecular characterization and diagnosis of QoI resistance in cucumber and eggplant fungal pathogens. Phytopathology 97:1458-1466.

Johnson, H. W. 1958. Registration of soybean varieties, VI; Lee (Reg. No. 23). Agron. J. 50:690.

Johnson, H. W. 1960. Registration of soybean varieties, VII; Hood (Reg. No. 30). Agron. J. 52:659.

Kim, H., Newell, A. D., Cota-Sieckmeyer, R. G., Rupe, J. C., Fakhoury, A. M., and Bluhm, B. H. 2013. Mating-type distribution and genetic diversity of Cercospora sojina populations on soybean from Arkansas: evidence for potential sexual reproduction. Phytopathology 103:1045-1051.
Mengistu, A., Bond, J., Mian, R., Nelson, R., Shannon, G., and Wrather, A. 2012 Resistance to frogeye leaf spot in selected soybean accessions in MG I through MG VI. Plant Health Prog. 13:13.

Mengistu, A., Young, H. K., Bellaloui, N., Arelli, P. R., Reddy, K. N., and Wrather, A. J. 2014. Tillage, fungicide, and cultivar effects on frogeye leaf spot severity and yield in soybean. Plant Dis. 98:1476-1484.

Mian, M. A. R., Missaoui, A. M., Walker, D. R., Phillips, D. V., and Boerma, H. R. 2008. Frogeye leaf spot of soybean: A review and proposed race designations for isolates of Cercospora sojina Hara. Crop Sci. 48:14-24.

Niblack, T. L., Arelli, P. R., Noel, G. R., Opperman, C. H., Orf, J. H., Schmitt, D. P., et al. 2002. A revised classification scheme for genetically diverse populations of Heterodera glycines. J. Nematol. 34:279-288.

Phillips, D. 1999. Frogeye leaf spot. Pages 20-21 in: Compendium of Soybean Diseases, 4th ed. American Phytopathological Society Press, St Paul, MN.

Phillips, D. V., and Boerma, H. R. 1981. Cercospora sojina race 5: A threat to soybean in the southeastern United States. Phytopathology 71:334-336.

Phillips, D. V., and Boerma, H. R. 1982. Two genes for resistance to race 5 of Cercospora sojina in soybeans. Phytopathology 72:764-766.

Probst, A. H., and Athow, K. L. 1964. Registration of Kent soybean. Crop Sci. 4: 240.

Ross, J. T. 1968. Additional physiologic races of Cercospora sojina on soybean in North Carolina. Phytopathology 58:708-709.

Shrestha, K. S., Cochran, A., Mengistu, A., Lamour, K., Rocha, A. C., and Young, H. K. 2017. Genetic diversity, QoI fungicide resistance, and mating type distribution of Cercospora sojina-Implications for the disease dynamics of frogeye leaf spot on soybean. PLoS One 12:e0177220.

Vanlerberghe, G. C. 2013. Alternative oxidase: A mitochondrial respiratory pathway to maintain metabolic and signaling homeostasis during abiotic and biotic stress in plants. Int. J. Mol. Sci. 14:6805-6847.

Villani, S. M., and Cox, K. D. 2014. Heteroplasmy of the cytochrome b gene in Venturia inaequalis and its involvement in quantitative and practical resistance to trifloxystrobin. Phytopathology 104:945-953.

Wallace, W. J. 1982. Introduction. Pages 1-2 in: Compendium of Soybean Diseases, 2nd ed. J. B. Sinclair, ed. American Phytopathological Society, St. Paul, MN.

Ward, J. H. 1963. Hierarchical grouping to optimize an objective function. Am. Stat. Assoc. J. 56:236-244.

Weiss, M. G. 1953a. Registration of soybean varieties, III; Lincoln (Reg. No. 5). Agron. J. 45:326-327.

Weiss, M. G. 1953b. Registration of soybean varieties, III; Blackhawk (Reg. No. 9). Agron. J. 45:328-329.

Weiss, M. G. 1953c. Registration of soybean varieties, IV; Ogden (Reg. No. 13). Agron. J. 45:571.

Zhang, G. R. 2012. Cercospora sojina: Over-winter survival and fungicide resistance. Graduate Diss., University of Illinois. http://hdl.handle.net/2142/31005.

Zhang, G. R., Allen, T. W., Bond, J. P., Fakhoury, A. M., Dorrance, A. E., Weber, L., et al. 2018. Widespread occurrence of quinone outside inhibitor fungicide-resistant isolates of Cercospora sojina, causal agent of frogeye leaf spot of soybean, in the United States. Plant Health Prog. 19:295-302.

Zhang, G. R., Newman, M. A., and Bradley, C. A. 2012a. First report of the soybean frogeye leaf spot fungus (Cercospora sojina) resistant to quinone outside inhibitor fungicides in North America. Plant Dis. 96:767.

Zhang, G. R., Pedersen, K. D., Phillips, D. V., and Bradley, C. A. 2012 b. Sensitivity of Cercospora sojina isolates to quinone outside inhibitor fungicides. Crop Prot. 40:63-68. 
ERRATUM / Volume 104, Number 2, 2020 / PDIS-02-19-0368-RE

In the article "Pathotype Grouping of Cercospora sojina Isolates on Soybean and Sensitivity to QoI Fungicides" by Alemu Mengistu, Jeffery D. Ray, Heather M. Kelly, Binbin Lin, Hao Yu, James R. Smith, Prakash R. Arelli, and Nacer Bellaloui, the captions for figures 3 and 4 were reversed. The correct figure-caption combinations are shown below:

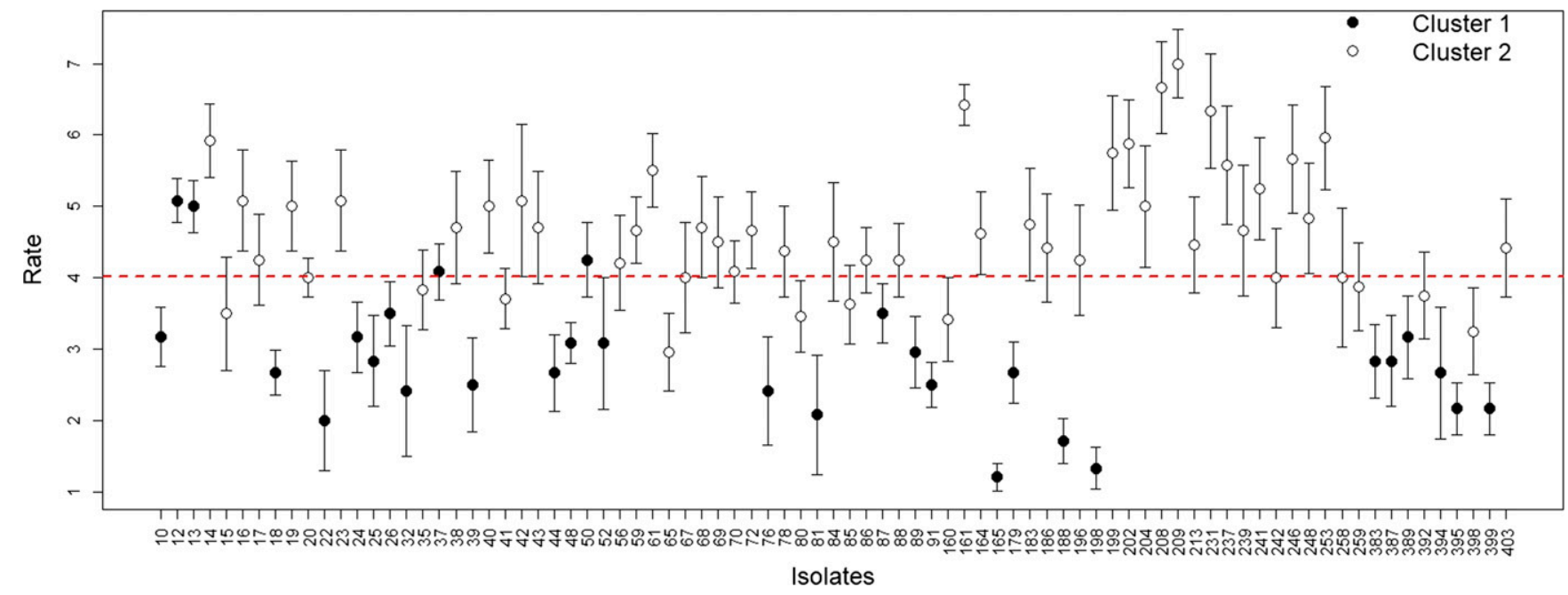

Fig. 3. Mean severity of each isolate of Cercospora sojina. The shaded circles indicate mean severity of Cluster 1 , whereas the open circles indicate mean severity of Cluster 2. The vertical lines indicate standard errors of each mean.

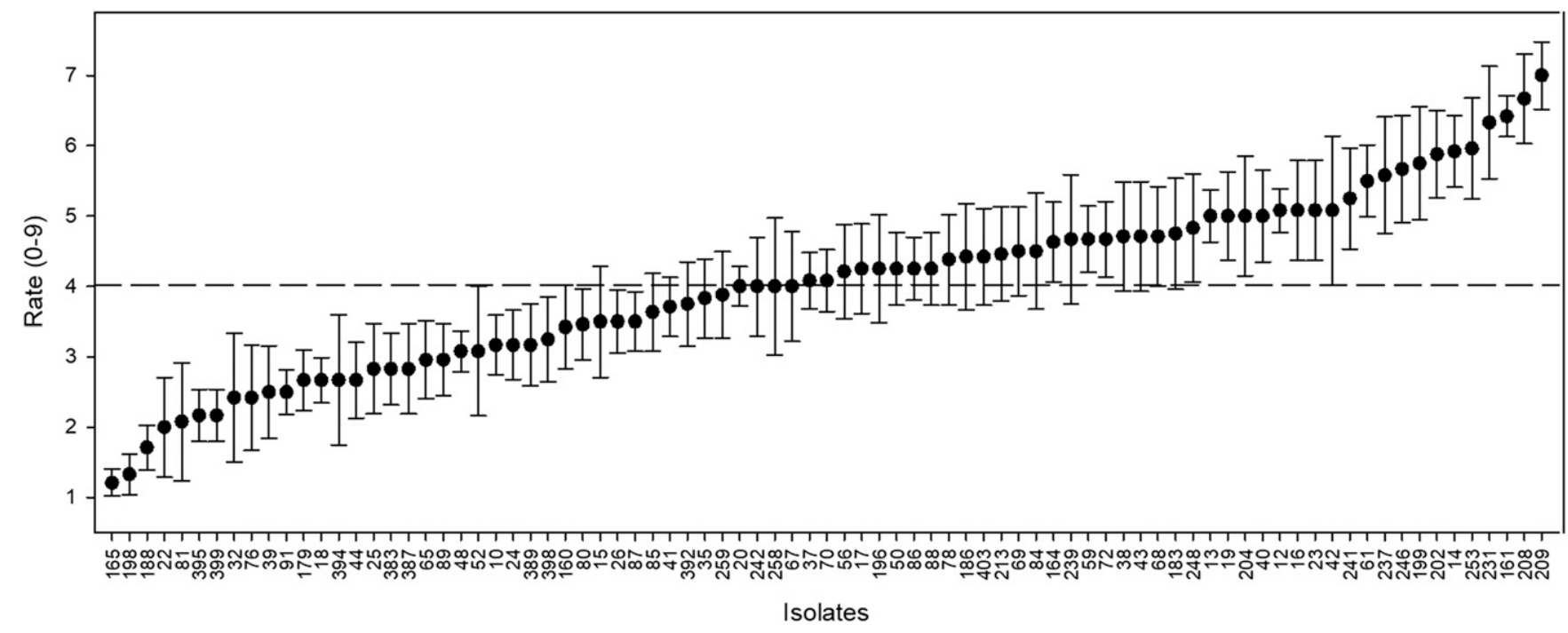

Fig. 4. Mean severity responses of the 83 isolates of Cercospora sojina across 12 soybean differentials ordered by isolate severity. The dots represent mean severity and the vertical lines indicate standard errors of each mean. 